\title{
A REMARK ON THE IMAGE OF THE AHLFORS FUNCTION
}

\author{
AKIRA YAMADA
}

\begin{abstract}
Let $\Omega$ denote a planar maximal region for bounded holomorphic functions and $p \in \Omega$. By an example we show that the complement in the unit disc of the image of the Ahlfors function for $\Omega$ and $p$ can be a fairly general set of logarithmic capacity zero.
\end{abstract}

1. Introduction. Let $\Omega$ be a planar region that supports nonconstant bounded holomorphic functions and let $p \in \Omega$. Set $B=\{f \mid f$ is holomorphic in $\Omega$ and $f(\Omega) \subset D\}$ where $D=\{z|| z \mid<1\}$. The Ahlfors function for $\Omega$ and $p$ is the unique function $F$ in $B$ such that

$$
F^{\prime}(p)=\max _{f \in B} \operatorname{Re} f^{\prime}(p) .
$$

It is elementary to show that $F(p)=0$. This paper is concerned with the image $F(\Omega)$ of the Ahlfors function.

Havinson [3] and Fisher [2] demonstrated that $D \backslash F(\Omega)$ has analytic capacity zero. We assume that the region $\Omega$ is maximal for bounded holomorphic functions in the sense of Rudin [8], since for nonmaximal regions the question about the size of $D \backslash F(\Omega)$ is trivial [5]. Recently, Minda [5] constructed an example of maximal regions showing that $D \backslash F(\Omega)$ can be a fairly general discrete subset of $D$. We shall extend Minda's result by showing that the image $F(\Omega)$ can omit a fairly general set of logarithmic capacity zero.

The author wishes to thank the referee for his helpful comments on the original version.

2. The example. The following construction is due to Minda [5]. Now we recall his basic construction in a slightly modified form.

Let $K$ be a compact subset of $D$ such that $\operatorname{Cap}(K)=0, K \cap \mathbf{R}=\varnothing$ and $\bar{K}=K$, where $\operatorname{Cap}(K)$ denotes the logarithmic capacity of $K$ and $\bar{K}$ the reflection of the set $K$ in the real axis. Set $\Delta=D \backslash K$ and $\Delta^{+}=\Delta \cap H$, where $H=\{z \mid \operatorname{Im} z>0\}$. Let $f$ : $H \rightarrow \Delta^{+}$be an analytic universal covering of the lower half-plane $\bar{H}$ onto $\Delta^{+}$. Let $\Gamma$ be the associated group of cover transformations. $\Gamma$ is a Fuchsian group of the second kind consisting of all Möbius transformations $T$ which map $H$ onto itself and satisfy $f \circ T=f$. Since $(-1,1)$ is a free boundary arc of $\Delta^{+}$, there is an open set $\sigma$ contained in the extended real line $\mathbf{R} \cup\{\infty\}$ such that $f$ extends continuously to

Received by the editors July 21, 1982 and, in revised form, September 13, 1982.

1980 Mathematics Subject Classification. Primary 30C75, 30C85.

Key words and phrases. Ahlfors function, logarithmic capacity. 
$H \cup \sigma$ and $f$ maps each component of $\sigma$ homeomorphically onto $(-1,1)$. Without loss of generality, we may assume that $\infty \in \sigma$ and that $f(\infty)=0$. Let $\sigma_{\infty}$ be the component of $\sigma$ that contains $\infty$. We extend $f$ to a holomorphic function on $\Omega=H \cup \sigma \cup \bar{H}$ by means of the Schwarz reflection principle: $f(\bar{z})=\overline{f(z)}$. We continue to denote the extended holomorphic function by $f$. It is elementary to verify that $f: \Omega \rightarrow \Delta$ is an analytic covering, that $f^{\prime}(\infty)>0$ and that the group of cover transformations associated with this covering is exactly $\Gamma$.

\section{The Ahlfors function of $\Omega$.}

LEMMA $1 . \Omega$ is a maximal region for bounded holomorphic functions.

Proof. See the proof of Proposition 1 in [5].

We are going to show that $f: \Omega \rightarrow \Delta$ is the Ahlfors function for $\Omega$ and $\infty$.

Let $E=\mathbf{R} \backslash \sigma$. Then $E$ is a compact subset of $\mathbf{R}$ and $T(E)=E$ for all $T \in \Gamma$. If $F$ denotes the Ahlfors function for $\Omega$ and $\infty$, a result of Pommerenke [6] implies that

$$
F(z)=\tanh g(z)
$$

where $g(z)=\frac{1}{4} \int_{E} d \zeta /(z-\zeta)$.

Let $L$ be the limit set of $\Gamma$ and denote by $m(L)$ its one-dimensional Lebesgue measure. Note that $L$ is closed and invariant under $\Gamma$.

LEMMA 2. $m(L)=0$.

Proof. Let $u(z)$ be the harmonic measure of $L$ in $H$. By the reflection principle we may assume that $u(z)$ is defined on $(\mathbf{C} \cup\{\infty\}) \backslash L$ and is harmonic there. Since $L$ is invariant under $\Gamma$ and $u(z)$ is bounded, $u(z)$ projects via the covering $f$ to a bounded harmonic function $U(z)$ in $\Delta=D \backslash K$. It follows from the assumption $\operatorname{Cap}(K)=0$ that $U(z)$ extends to a harmonic function on $D[9$, p. 261]. Since the boundary value $U\left(e^{i \theta}\right), 0 \leqslant \theta<2 \pi$, vanishes everywhere, we see that $U(z)=0$ in $D$. Thus $u(z)=0$ in $H$, which implies that $m(L)=0$.

Let $E^{\prime}=E \backslash L$ and let $E_{0}$ be a measurable fundamental set of $E^{\prime}$. Then $m\left(E^{\prime}\right)=$ $m(E)$ and $E^{\prime}=\cup_{T \in \Gamma} T\left(E_{0}\right)$ (disjoint union). Noting that $\Gamma$ is of convergence type and $\infty$ is an ordinary point, we have

$$
g(z)=\frac{1}{4} \int_{E^{\prime}} \frac{d \zeta}{z-\zeta}=\frac{1}{4} \int_{E_{0}} h(z, \zeta) d \zeta
$$

where the Poincaré series

$$
h(z, \zeta)=\sum_{T \in \Gamma} \frac{T^{\prime} \zeta}{z-T \zeta}
$$

converges uniformly and absolutely on compact subsets of $\mathbf{C} \backslash L$ after possibly omitting a finite number of terms. For $z \in \mathbf{C} \backslash L, h(z, \cdot)$ is a meromorphic automorphic form on $\mathbf{C} \backslash L$ of weight -2 for $\Gamma$ with at most simple poles at $\zeta \in \Gamma z$. For $\zeta \in \mathbf{C} \backslash L, h(\cdot, \zeta)$ is a meromorphic Eichler integral on $\mathbf{C} \backslash L$ of order 0 with at most simple poles at $z \in \Gamma \zeta[4$, p. 221]. Here we remark that in his book [4], Kra restricts himself to stating the above results only in the case of weight $-2 q$ with $q \geqslant 2$. It is, 
however, easily seen that if $\Gamma$ is a Fuchsian group of convergence type, these results continue to hold in our present case $q=1$.

Lemma 3. $h(T z, \zeta)=h(z, \zeta)$ for all $T \in \Gamma, z \in \mathbf{C} \backslash L$ and $\zeta \in \mathbf{C} \backslash L$.

Proof. First assume that $z \in H$ and $\zeta \in H$. Then we have the following identity: for all $\varphi \in A_{1}^{2}(\Gamma)$ and $T \in \Gamma$,

$$
\pi \int_{z}^{T z} \varphi d \zeta=\iint_{\omega} \varphi \cdot[\overline{h(T \bar{z}, \cdot)}-\overline{h(\bar{z}, \cdot)}] d \xi d \eta
$$

where $A_{1}^{2}(\Gamma)$ denotes the space of square integrable holomorphic automorphic forms of weight -2 for $\Gamma$ and $\omega$ is a fundamental domain for $\Gamma$ in $H$ whose boundary has (two-dimensional) Lebesgue measure zero. We remark that $A_{1}^{2}(\Gamma)$ is a Hilbert space and $h(\bar{T} z, \cdot)-h(\bar{z}, \cdot)$ belongs to $A_{1}^{2}(\Gamma)$. The identity (3) is a variant of the one obtained in the work of Rao [7, Theorem 2] and is proved analogously. Hence we omit the proof. On the other hand, by using the fact that if $K$ is a compact subset of C with logarithmic capacity zero and $U$ is an open set with $K \subset U$, then every analytic function in $A L^{2}(U \backslash K)$ has an analytic extension to $U$ [1, p. 483], we have the isometries

$$
A_{1}^{2}(\Gamma) \cong A L^{2}\left(\Delta^{+}\right) \cong A L^{2}(D \cap H)
$$

where $A L^{2}(X)$ denotes the space of square integrable holomorphic functions on $X$. Note that all periods of any function $\varphi \in A L^{2}(D \cap H)$ vanish, since $D \cap H$ is simply-connected. Observing that the left side of (3) represents a "period" along the loop associated to $T \in \Gamma$, we find that $h(T \bar{z}, \cdot)-h(\bar{z}, \cdot)$ is orthogonal to $A_{1}^{2}(\Gamma)$, concluding that for $z \in \bar{H}$ and $\zeta \in H, h(T z, \zeta)=h(z, \zeta)$. Analytic continuation now yields Lemma 3.

It is clear from the above lemma, (1) and (2) that the Ahlfors function $F(z)$ satisfies

$$
F \circ T=F \quad \text { for all } T \in \Gamma .
$$

Proposition. The analytic covering projection $f: \Omega \rightarrow \Delta$ is the Ahlfors function for $\Omega$ and $\infty$.

Proof. Lemma 3 shows that the Ahlfors function $F$ is invariant under the group $\Gamma$. This easily implies that $F$ induces a holomorphic function $\tilde{F}: \Delta \rightarrow D$ such that $F=\tilde{F} \circ f$ and $\tilde{F}(0)=0$. Since the condition $\operatorname{Cap}(K)=0$ implies that $K$ has analytic capacity zero, the bounded holomorphic function $\tilde{F}$ has an extension which is a holomorphic self-map of $D\left[9\right.$, p. 261]. Hence Schwarz's lemma yields that $F^{\prime}(\infty)=$ $\tilde{F}^{\prime}(0) f^{\prime}(\infty) \leqslant f^{\prime}(\infty)$. On the other hand it is clear that $F^{\prime}(\infty) \geqslant f^{\prime}(\infty)$. This gives $f=F$ since the Ahlfors function is unique.

REMARK. It is still an open question whether the Ahlfors function for a maximal planar region can actually omit a set of analytic capacity zero.

\section{REFERENCES}

1. S. Axler, J. B. Conway and G. Mcdonald, Toeplitz operators on Bergman spaces, Canad. J. Math. 34 (1982), 466-483.

2. S. D. Fisher, On Schwarz's lemma and inner functions, Trans. Amer. Math. Soc. 138 (1969), 229-240. 
3. S. Ya. Havinson, Analytic capacity of sets, joint nontriviality of various classes of analytic functions and the Schwarz lemma in arbitrary domains, Amer. Math. Soc. Transl. (2) 43 (1964), 215-266.

4. I. Kra, Automorphic forms and Kleinian groups, Benjamin, Reading, Mass., 1972.

5. C. D. Minda, The image of the Ahlfors function, Proc. Amer. Math. Soc. 83 (1981), 751-756.

6. Ch. Pommerenke, Über die analytische Kapazität, Arch. Math. (Basel) 11 (1960), 270-277.

7. K. V. R. Rao, Reproducing formulas for Poincaré series of dimension -2 and applications, Ann. of Math. Studies, No. 66, Princeton Univ. Press, Princeton, N. J., 1971, pp. 329-340.

8. W. Rudin, Some theorems on bounded analytic functions, Trans. Amer. Math. Soc. 78 (1955), 333-342.

9. L. Sario and K. Oikawa, Capacity functions, Springer-Verlag, Berlin, Heidelberg and New York, 1969.

Department of Mathematics, Tokyo Institute of Technology, Ohokayama, Meguro, Tokyo, 152 , JAPAN 